\title{
Sentido de Vida na Fase Adulta e Velhice
}

\author{
Meaning of Life in Adulthood and Later Life
}

\author{
Cinara Sommerhalder* \\ Universidade Estadual de Campinas
}

\begin{abstract}
Resumo
A busca incessante de um sentido para a vida faz parte dos questionamentos existências. Viktor Frankl foi pioneiro em abordar o tema na Psicologia. Posteriormente, a pesquisa fez uso de seus ensinamentos e ampliou a definição do construto. Estudos transculturais têm contribuído para uma melhor compreensão do tema, mas as pesquisas ainda precisam ser aprofundadas. Embora os instrumentos disponíveis ainda estejam sendo testados e aperfeiçoados, as informações já alcançadas representam uma contribuição de grande relevância para o estudo do desenvolvimento do adulto e da velhice. Este artigo objetiva mostrar a evolução do conceito e como as pesquisas vêm tratando o assunto na área do desenvolvimento do adulto e da velhice.

Palavras-chave: Sentido de vida; Desenvolvimento adulto; Velhice.
\end{abstract}

\begin{abstract}
The unceasing search for meaning to life is part of the existential questioning. Viktor Frankl is a pioneer in this field. Later, researchers have used his lessons to expand the definition of the construct 'meaning of life'. Transcultural studies lead to a better understanding of this theme, but further research still needs to be done. The available instruments are being tested and improved; however, we already have relevant information on the theme, and it contributes largely to the study of the development of adulthood and later life. This article aims at showing the evolution of the concept 'meaning of life' and how researches have been dealing with the theme in the field of adulthood and later life development.

Keywords: Meaning of life; Adulthood; Later life.
\end{abstract}

O sentido de vida faz parte dos questionamentos existenciais e é uma busca constante do ser humano. A Psicologia tem uma longa trajetória de dedicação à compreensão do conceito para melhor explicitá-lo e, assim, compreender sua relação com os mecanismos de ajustamento e adaptação, contribuindo para o estudo do desenvolvimento humano e do envelhecimento.

Esta pesquisa teve como objetivo realizar um levantamento bibliográfico exploratório para buscar: as definições de sentido de vida, a evolução do conceito e as linhas de pesquisas, como as pesquisas estão sendo conduzidas e de que forma essas descobertas podem contribuir para a Psicologia do Desenvolvimento no que tange à compreensão dos mecanismos de ajustamento e adaptação na vida adulta e na velhice. As bases de dados consultadas foram: AgeLine, PsycoInfo, Lilacs e MedLine, com as palavras-chave: meaning of life, meaning in life, personal meaning e personal meaning sources.

Foram encontrados dois grandes grupos de estudos. O primeiro relaciona sentido de vida a aspectos de saúde, que compreendem doenças graves como Aids e câncer,

* Endereço para correspondência: Rua Valentim Trevisan, 686 Jd. Janorama, Vinhedo, SP, Brasil, CEP 13280-000. Email: cinarasommer@hotmail.com iminência de morte, saúde mental e saúde física, e a aspectos psicológicos como estratégias de enfrentamento, afetividade, sentimentos positivos, traços de personalidade, motivação, redes de suporte, bem-estar, formação da identidade de crianças e adolescentes, responsabilidade, ajustamento psicológico diante de perdas significativas, religiosidade e espiritualidade. Os modelos teóricos estão pautados em teorias de estresse, enfrentamento, nos pressupostos de Viktor Frankl, em teorias de controle, de bem-estar, enfrentamento religioso/espiritual e depressão. O segundo grupo é mais específico, investiga as fontes de sentido na vida. Aqui, foram encontrados estudos que definem o construto e trabalham com um modelo teórico baseado nessa definição. Essa linha de pesquisa investe em estudos transculturais e construção e validação de instrumentos.

Com base nessas informações, serão apresentados relatos de pesquisas dos dois grandes grupos de investigações e as lacunas que ainda precisam ser preenchidas.

\section{Definição do Conceito: De Viktor Frankl aos Dias Atuais}

Na Psicologia, Viktor Frankl (1905-1997) foi pioneiro ao escrever e questionar sistematicamente sobre o sentido da vida. Frankl dedicou-se ao trabalho clínico-tera- 
pêutico e fundou a Logoterapia, também conhecida como Terceira Escola Vienense de Psicoterapia, ou Psicologia do Sentido da Vida, cuja premissa básica é a busca de sentido para a vida. O trabalho de Frankl é todo voltado às questões existenciais.

De acordo com o autor, não é possível ao psicólogo dar um sentido para a vida do outro, mas sim ajudá-lo a encontrar o seu próprio sentido (Sommerhalder \& Goldstein, 2006). Ele propõe quatro fatores que podem levar a pessoa a encontrar um sentido para a vida: (a) $A$ valorização do que é importante para a pessoa, ou seja, aquilo que teve significado durante a vida, desde os pequenos até os grandes eventos. As experiências de vida influenciam na forma que cada um tem de lidar com as situações. (b) As escolhas - o indivíduo é responsável por cada escolha que faz ao longo da vida, inclusive diante de situações adversas. Frankl aborda o sofrimento como uma grande oportunidade de crescimento pessoal, que, no entanto, depende de como a pessoa o enfrenta. Ela pode sucumbir à dor, ou extrair ensinamentos da situação difícil. (c) Responsabilidade - por tudo o que a pessoa faz, pelas escolhas e decisões. (d) Significado imediato - dar sentido às coisas que acontecem na vida diária, tanto as experiências positivas, quanto as negativas (Frankl, 1999).

Para uma vida com sentido, três valores são significativos: valor criativo - produzir algo significativo, fazer uma boa ação; valor vivencial - vivenciar, experimentar aquilo que a pessoa recebe do mundo, que pode estar relacionado às experiências de trocas afetivas ou mesmo interagindo com os objetos do mundo. $\mathrm{O}$ sentido pode ser encontrado em uma experiência independente de qualquer ação, e um único momento de experiência intensa pode prover significado para a vida toda; valor atitudinal - transformar a tragédia pessoal em triunfo, ou seja, buscar lições de crescimento pessoal nos momentos difíceis (Frankl, 1999). Segundo esse autor, a falta de sentido para a vida poderia desencadear sintomas como ansiedade, depressão, falta de esperança e declínio físico.

Frankl contribuiu enormemente na área clínica. Já na área de pesquisa, outros autores somaram novas dimensões ao construto. Reker e Wong (1988) definem sentido de vida como um construto multidimensional, composto por um componente cognitivo, englobando crenças e interpretações do mundo. Isso ajuda o indivíduo nas suas preocupações existenciais, na organização e na compreensão das experiências. Há também um componente motivacional, que reúne os sistemas de valores de cada indivíduo, os quais interferem na realização das metas pessoais. Por último, há um componente afetivo, relacionado ao sentimento de satisfação, que é a convicção de que a vida vale a pena. As experiências pessoais medeiam essas escolhas.

Reker (2000) observa que o construto sentido de vida tem sido considerado, nos últimos anos, o mais complexo e discutido na literatura, por diferentes abordagens. As dimensões mais exploradas levam em conta o modo como o sentido é experienciado. A literatura denomina componentes estruturais, que representam aquilo que as pessoas relatam das suas vivências, como as percebem. Eles estão relacionados às experiências em si. É também nessa perspectiva que se inserem as fontes de sentido, ou seja, em quais esferas da vida se localizam as razões do viver.

Reker (1997) afirma que o sentido de vida está associado a ter um propósito, uma direção, uma razão para a existência, ter uma percepção de identidade pessoal e interesse social, além de sentir-se satisfeito com a vida, mesmo diante de situações difíceis, quando o "para que viver" é essencial. Na dimensão individual, as crenças, os valores e as necessidades da pessoa norteiam quais as metas que ela deve perseguir e em quais relacionamentos deve investir. Isso funciona como um guia para as buscas e os engajamentos individuais.

O sentido de vida - que compreende esquemas conceituais, categorias do self e interpretação da vida orienta o indivíduo em direção ao viver. É uma rede cognitivo-afetiva que abrange metas, comportamentos e várias classes de padrões de autoavaliação. É direcionado pelos valores da vida e está associado à saúde mental (Prager, 1997).

O construto ainda é considerado novo na área de estudos do envelhecimento. O eixo desses trabalhos é o conceito de envelhecimento como um processo universal e, como tal, envolvido por indagações sobre a continuidade do ser. Um dos questionamentos mais comuns é "por que estou aqui; para onde vou?". Apesar disso, é preciso lembrar que, salvo as semelhanças inerentes à espécie, adultos e pessoas idosas são indivíduos com histórias de vida, aspirações e atribuições de significado para suas existências que corroboram suas vivências, ou seja, envelhecer é uma experiência singular, ligada à trajetória pessoal, o que define sua particularidade. Prager (1997) alerta para a necessidade de se pensar a velhice não como uma categoria etária universal, mas sim como uma fase na qual a singularidade dos indivíduos deve ser ponto inicial de qualquer trabalho.

Encontrar sentido está relacionado a um equilíbrio entre perdas e ganhos, dar significado para as atitudes e os eventos cotidianos e ter um propósito na vida. Vários fatores influenciam a percepção de sentido na vida. Os fatores internos, que estão ligados ao desenvolvimento do indivíduo, podem ser: personalidade, estratégias de enfrentamento, religiosidade, espiritualidade, sentimento de pertencimento, história de vida; já os fatores externos, que pertencem ao meio e corroboram o significado que as pessoas dão à vida, relacionam-se a: oportunidades sociais, trabalho, renda, lazer, suprimento das necessidades básicas de sobrevivência e segurança.

Encontrar sentido para as experiências da vida, estar no mundo com um objetivo, em prol de algo, conseguir dar um propósito para as atividades diárias e até mesmo encontrar significado para as dificuldades são alguns dos caminhos para dar sentido à vida. 
De Vogler e Ebersole (1980) conceituam sentido de vida em oito dimensões: compreensão (busca de mais conhecimento); relacionamento (orientação interpessoal); serviço (ajudar, dar orientação); crença (acreditar em algo); expressão (artística, esportiva, musical, literária); conquistas (respeito, posses, responsabilidade); crescimento (desenvolvimento do potencial pessoal, cumprimento de metas) e existencial-hedonística (a importância do prazer na vida diária). Paralelamente, há as metas de vida, realização e trabalho (incluindo recompensa econômica, sucesso, status social e satisfação), boas relações pessoais, metas filosóficas e religiosas, serviço social, ausência de dificuldade (segurança, automanutenção, saúde, conforto), satisfação e crescimento pessoal (incluindo aprendizagem, conhecimento e domínio).

Fiske e Chiriboga (1991) relatam que a dimensão $\mathrm{cres}$ cimento pessoal é a mais citada nos estudos sobre mudança e continuidade ao longo da vida, que são temas diretamente ligados ao sentido na vida e de interesse da Psicologia do Desenvolvimento. Guttman e Huyck (1994) afirmam que o envelhecimento não é só a continuidade de uma mudança, mas também um tempo de adaptação às perdas. Assim, encontrar um sentido para a vida e para as experiências ajuda a enfrentar as perdas, que aumentam com o avançar da idade.

Baumeister (1991) define o sentido de vida como uma representação mental de possíveis relações entre coisas, eventos e relacionamentos, ou seja, a importância que as pessoas dão às pequenas e às grandes coisas da vida. Thompson e Janigiana (1988) afirmam que o indivíduo tem esquemas de vida que lhe conferem ordem, propósito e direção. Kaufman (1987) considera que a vida não tem um sentido por inteiro, sustentando a ideia de fragmentos ou histórias interessantes, atividades significativas, investimento e realização de metas. Essa posição também é defendida pelos existencialistas, que argumentam que cada pessoa constrói ativamente o significado de sua vida. De Vogler e Ebersole (1980) ressaltam que o relacionamento com a família e com os amigos está diretamente ligado ao sentido de vida. Krause (2007) afirma que o significado na vida dos idosos está associado à natureza do suporte social que eles recebem de familiares e amigos próximos. Em revisão do tema, Sommerhalder e Erbolato (2008) analisam pesquisas significativas que apontam os relacionamentos mais íntimos com amigos e familiares como importantes componentes do sentido de vida.

O construto sentido de vida tem uma dimensão individual, como também tem um componente cultural. Os valores e temas da vida, embora sejam individuais, fazem parte de um todo maior que é a cultura na qual o sujeito está inserido, e isso influencia as decisões pessoais, ou seja, os sujeitos decidem também com base em opiniões, valores e metas coletivas (Prager, 1997). A necessidade de estar inserido no contexto social, de sentir-se parte da cultura faz com que muitos fatores re- lacionados ao sentido individual sejam compartilhados pela coletividade.

Há teóricos que defendem que o sentido de vida não muda muito ao longo da existência, ou seja, sofre somente transformações graduais na conjunção com mudanças nos sistemas de crenças e valores (Zika \& Chamberlain, 1992). Envelhecer é um processo e as preferências não mudam drasticamente, ou seja, elas acompanham esse caminho. As escolhas vão sendo feitas tendo por base as crenças, os valores e as experiências do indivíduo e, salvo em situações extraordinárias - como doenças graves, catástrofes etc., que conduzem o indivíduo a uma ressignificação da sua vida que, eventualmente, o impulsionam a mudanças drásticas -, o padrão de comportamento e das escolhas acompanha o desenvolvimento.

Atchley (1989) desenvolve um modelo que trata de mudanças sofridas por adultos. A premissa central de sua tese, chamada de teoria da continuidade, é a de que na meia-idade e na velhice as mudanças sofridas pelos adultos têm por finalidade a adaptação, a preservação e a manutenção de estruturas externas e internas, e defende que, para tanto, são utilizadas estratégias ampliadas, desenvolvidas e adaptadas às novas situações. Os adultos utilizam-se das experiências passadas para resolver as questões do presente, numa forma de continuidade e adaptação. Coerência e consistência são parte desse pressuposto, o que não significa oposição à mudança. Crescimento, desenvolvimento e mudanças adaptativas não implicam permanecer sempre da mesma forma. Acontecimentos variados podem modificar a direção e o contexto de vida, o que não significa rompimento total com o passado. $\mathrm{O}$ modelo consiste de princípios gerais adaptativos para as pessoas que estão envelhecendo normalmente e de formas de utilização dessas estratégias no trabalho, na vida pessoal, na esfera familiar e no convívio social.

Os resultados positivos do envelhecimento normal ocorrem quando os indivíduos usam estratégias de adaptação para as mudanças associadas ao processo. Apesar das mudanças nas estruturas de identidade, na meiaidade muitos pontos resultam da continuidade e da estabilidade de aspectos globais do self e da identidade (Atchley, 1989).

O modelo de continuidade liga adaptação à história de vida, e estabilidade autopercebida (continuidade interna) à rede de suporte social e à interação na comunidade (continuidade externa). Continuidade interna é essencial para a integridade do ego e necessária para a autoestima. Kaufman (1987) afirma que adaptação na vida tardia é um processo em que passado e presente estão em interação; para isso, o passado precisa ser organizado e compreendido como forma de auxílio para o enfrentamento dos eventos presentes. A interação social é de suma importância nesse processo e, ainda segundo o autor, se conseguíssemos descobrir as fontes de sentido que estão 
imbricadas nesse contexto, teríamos informações valiosas a respeito de desenvolvimento e continuidade. Lazarus (1998) também aborda que envelhecer bem requer compensação de perdas físicas e mentais, com o objetivo de manter em destaque os valores importantes e as metas de vida.

As pessoas mais velhas necessitam manter o senso de continuidade com o passado, porque isso ajuda no enfrentamento das mudanças. Os estudos apontam para a continuidade do sentido na vida e continuidade do self.

\section{Revisão de Literatura}

Pesquisando em bases de dados como AgeLine, PsycoInfo, Lilacs e MedLine, observou-se que há dois grandes grupos de pesquisas na literatura: o primeiro reúne estudos que associam sentido de vida a aspectos de saúde, entre os quais doenças graves como Aids e câncer, saúde mental, saúde física e iminência de morte, e a aspectos psicológicos, como estratégias de enfrentamento, afetividade, sentimentos positivos, traços de personalidade, motivação, redes de suporte, bem-estar, formação da identidade de crianças e adolescentes, responsabilidade, ajustamento psicológico diante de perdas significativas, inclusive morte, viuvez, asilamento, religiosidade, espiritualidade. Nessa linha há pesquisadores brasileiros, como Calache (1993), Diniz (1992), Huf (1999), Leonardi (2007), Vitola (1998) e Zaleski (1996, 2001), e pesquisadores internacionais, como Davis, NolenHoeksema e Larson (1998), Fry (1998, 2001), Kirby, Coleman e Daley (2004), Krause (2003, 2004), Prager (1996, 1997), Reker (1997), Reker e Wong (1988), Wong (1998). Esses estudos fundamentam-se nos pressupostos de Viktor Frankl, em teorias de enfrentamento, de estresse, de controle, de bem-estar, enfrentamento religioso/espiritual e depressão. Alguns relatos não investigaram especificamente o sentido de vida nem utilizaram modelos teóricos que englobassem o tema, mas, como abordam aspectos importantes da vida adulta e do envelhecer bem, seus resultados podem ser usados como exemplos, pois nos dão pistas sobre pontos essenciais que dão significado à vida.

O segundo grupo de pesquisas enfoca especificamente as fontes de sentido na vida, ou seja, a que esferas os indivíduos atribuem sentido em suas vidas. Nessa linha, o que mais encontramos são relatos de pesquisas transculturais, que buscam as similaridades das fontes de sentido na vida adulta e na velhice. Os pesquisadores procuram identificar aspectos que podem ser comuns no desenvolvimento. Essa linha utiliza modelos teóricos de sentido de vida e investe em validação e construção de instrumentos de medida.

A seguir serão expostos dados de pesquisas, para uma visão geral de como as investigações estão sendo conduzidas nos dois grupos.

Halama (2000) buscou as relações entre sentido de vida, estratégias de enfrentamento e sentimento de frustração em adolescentes eslováquios. Sua tese baseou-se na hipótese de que o sentido na vida poderia ser um importante auxiliar nas estratégias positivas de enfrentamento e manejo do estresse. Os resultados apontaram relação significativa entre o sentimento de vazio existencial e reações agressivas. Também indicaram que a falta de sentido - representada por tédio, apatia e sentimento de indiferença - pode diminuir a habilidade de enfrentamento e, consequentemente, aumentar a frustração, que tem relação significante com a agressividade. Em síntese, a pesquisa revela relação estreita entre falta de sentido de vida e agressividade.

Estudos com populações idosas mostram a relevância do sentido como fator protetor para depressão. Reker (1997), num estudo sobre depressão em idosos, revela que ter um propósito e ser otimista são fatores de proteção contra a depressão. Fry (2001), em uma pesquisa com adultos viúvos, com o objetivo de investigar se variáveis existenciais como o significado pessoal, o otimismo, a religiosidade e a acessibilidade ao suporte religioso eram preditores de bem-estar psicológico, relata que ter um propósito na vida ajuda a superar a perda e também na prevenção de depressão. Krause (2003) investigou o significado religioso, definido como um processo que envolve a religião como esforço para descobrir um senso de propósito na vida, um senso de direção e uma razão para a existência, em idosos. O autor associou significado religioso a bem-estar subjetivo. Concluiu que os idosos que tinham o senso de significado religioso tenderam a ter maior nível de satisfação na vida, maior autoestima e otimismo.

$\mathrm{Na}$ área da saúde, Kahana e Kahana (2001) investigaram o sucesso do envelhecimento de pacientes com HIV/ Aids. Eles sugerem que aspectos do envelhecimento bemsucedido - como esperança, altruísmo, autoestima, estratégias de enfrentamento e satisfação com a vida podem interferir na percepção de sentido de vida e, consequentemente, na qualidade de vida dos sujeitos. No Brasil, Zaleski (1996) examinou aspectos do sentido de vida em soropositivos adultos para HIV, valendo-se da tríade trágica proposta por Frankl: sofrimento, sentimento de culpa e morte. Família, amigos, esperança de cura, religião e amor fundamentaram o sentido de vida dessas pessoas. Posteriormente a autora pesquisou o significado da culpa existencial em soropositivos para HIV (Zaleski, 2001). Encontrou relações importantes entre o sentimento de culpa e a condição que a doença traz aos filhos. Familiares e amigos foram importantes fontes de suporte e enfrentamento da culpa. Leonardi (2007) enfocou o sentido de vida num grupo de portadores de transtornos mentais e encontrou dados importantes relacionando a relevância da solidariedade como fonte de sentido para um existir pleno.

Esses estudos mostram que as diferentes gerações percebem o sentido de vida de formas variadas, mas há pontos que são comuns, como as necessidades básicas de comer, de se abrigar e de segurança, necessidade de 
lazer, trabalho criativo, relacionamento pessoal, realização e crescimento pessoal e social, ativismo político, altruísmo, valores tolerantes e ideais, tradição, cultura e religião (Reker, Peacock, \& Wong, 1987).

As diferenças na percepção de um sentido para a vida, ao longo dos anos, estão associadas ao grau de consciência de mundo que cada um experimenta em diversos momentos da vida. Variáveis como gênero, idade, nível educacional, papel social desempenhado em um dado momento, profissão, religiosidade e espiritualidade, história de vida, fatores de personalidade e apoio social devem ser mais bem investigados, porque elas interferem na percepção de sentido de vida. Talvez os idosos constituam um grupo que tem predisposição para perceber a vida com mais sentido e propósito do que adultos de meia-idade e pessoas mais jovens. Isso porque eles são mais realistas em relação à persecução de metas e têm menor predisposição ao sentimento de vazio existencial. Os idosos tendem a fazer uma leitura mais fiel da realidade e de suas capacidades, o que os encaminha para decisões mais acessíveis, realizáveis, compatíveis com a situação e a finalização efetiva da tarefa. Desse modo, ficam menos expostos ao sentimento de frustração (Reker, 2001).

Em relação às fontes de sentido na vida, os primeiros estudos mostravam que as fontes de sentido na vida estavam ligadas a crescimento pessoal, relacionamentos, religião, metas filosófico-espirituais, sucesso, patrimônio, ganhos em sabedoria, altruísmo, ações hedonistas, realização profissional e segurança (De Vogler \& Ebersole, 1980; Fiske \& Chiriboga, 1991; Thurner, 1975). Baseado nessas informações, na década de 1990 Reker organizou tais dados e elaborou um instrumento de medida específico na investigação das fontes de sentido, o Sources of Personal Meaning Profile - Revised ([SOMPR], Reker, 1996), que foi muito utilizado em estudos transculturais. É uma escala tipo Likert, com 17 itens avaliados num continuum de sete pontos e suas respostas vão de "não muito significativo para mim" (1) a "extremamente significativo para mim" (7), podendo ser autoaplicada. Os itens da escala são: participação em atividades de lazer, satisfação de necessidades básicas do dia a dia, participar de atividades criativas, envolvimento em relações pessoais e com familiares e/ou amigos, reconhecimento por aquisições pessoais, experiência pessoal de crescimento, participação em atividades religiosas, interesse em causas sociais, estar a serviço do outro, preservação de valores humanos e ideais, preservação da cultura e da tradição, deixar legado para as próximas gerações, sentimento de segurança financeira, interesse em direitos humanos, participação em atividades hedonistas (jogos, festas), aquisição de posses materiais para poder gozar de uma boa vida e relacionamento com a natureza. O objetivo do SOMP era buscar as similaridades das fontes de sentido que poderiam fazer parte do desenvolvimento humano.
Tais estudos transculturais focavam o desenvolvimento do adulto e tinham por objetivo trabalhar com grupos nas três faixas etárias: adulto jovem, meia-idade e idosos, de ambos os gêneros, e comparar os resultados entre as diferentes faixas etárias e entre diferentes nacionalidades. A seguir, são apresentados os principais resultados desses estudos que utilizaram o SOMP-R como instrumento de pesquisa. Reker e Wong (1988) trabalharam com adultos canadenses nas três faixas etárias. Para todos os grupos encontrou similaridade nas seguintes fontes: relacionamento pessoal, satisfação de necessidades básicas, crescimento pessoal, atividades de lazer, preservação de valores e ideais, realização pessoal e altruísmo. $\mathrm{O}$ autor concluiu que há uma mudança qualitativa na velhice, ou seja, há uma tendência no desenvolvimento em direção a um processo mais interiorizado, egocêntrico, autopreocupado, ou seja, o indivíduo se volta progressivamente para o controle e a satisfação das suas necessidades básicas. Esse processo de interiorização, expressivo a partir da segunda metade da vida, pode influenciar nas fontes de sentido na vida.

Numa outra tentativa de investigar fatores culturais ligados às fontes de sentido na vida, Prager (1996) compara os dados de seu estudo - conduzido com adultos australianos nas três faixas etárias - aos de Reker e Wong (1988), que trabalhou com os mesmos grupos etários, mas com amostra de canadenses. Como no estudo de Reker e Wong (1988), a hipótese de tendência à interioridade no envelhecimento também foi considerada por Prager (1996). Segundo este autor, se ela fosse verdadeira, os resultados de sua pesquisa com a amostra australiana deveriam ser muito próximos aos achados da amostra canadense de Reker e Wong (1988), uma vez que a interiorização faria parte do desenvolvimento do adulto e não seria apenas um fator específico de grupos particulares.

Os resultados apontaram duas fontes de sentido como sendo importantes para as duas amostras e para todas as faixas etárias: satisfação de necessidades básicas e relações pessoais. Preservação de valores e ideais humanos também foram fontes importantes para os grupos. Esses resultados indicaram os pontos de similaridade nas fontes de sentido nos diferentes grupos etários e culturais, o que sugere que algumas fontes podem ser comuns aos indivíduos adultos.

Posteriormente Prager (1997) comparou os dados desse estudo australiano com dados de uma amostra israelense, todos indivíduos adultos, pertencentes às três faixas etárias e de ambos os gêneros. Concluiu que houve similaridade na atribuição de sentido entre os grupos etários das diferentes nacionalidades, tanto quanto na percepção de sentido entre os diferentes grupos etários quando comparados entre si e entre as amostras das diferentes nacionalidades. As mulheres idosas deram mais ênfase a conceitos humanistas, sociais e culturais, corroborando outras descobertas (por exemplo, Reker \& Wong, 
1988) e apontando para uma constância da importância da realização individual, com o envelhecimento, e um aumento na atribuição de sentido para a ética social e respeito ao outro, incluindo a família. Tanto para as mulheres israelenses quanto para as australianas, o envolvimento em relações pessoais foi a mais importante fonte de sentido.

Essas descobertas evidenciaram uma diversificação nas fontes de sentido na vida entre todos os grupos etários; porém, foram encontradas particularidades entre os grupos, além da constatação de que determinadas fontes estão relacionadas com a idade. Todos os grupos atribuíram grande importância a relacionamento pessoal, preocupação humanista e participação em atividades de lazer. Os grupos mais velhos foram unânimes em relação à importância de preservar valores e ideais humanos. Os homens mais velhos deram grande importância à segurança financeira e os grupos mais jovens enfocaram experiências de crescimento pessoal. As fontes de sentido que refletem transcendência do self foram significativamente mais importantes para os grupos de homens mais velhos, quando comparados com os jovens. Para os jovens, as fontes ligadas ao self foram as mais importantes (experiência de crescimento pessoal, deixar legado para as próximas gerações e participação em atividades hedonistas como jogos, festas etc.). Fontes auto-orientadas, como realização pessoal, foram mais relevantes para os grupos mais jovens. Os homens mais velhos demonstraram mais interesses em áreas de sentido que transcendiam o self, do que naquelas que refletiam preocupação com o self, com exceção da satisfação de necessidades básicas.

As informações obtidas nos estudos de Prager (1996, 1997) e Reker e Wong (1988) permitem afirmar que as fontes de sentido relacionamento pessoal e satisfação de necessidades básicas são comuns aos indivíduos adultos, mas não se pode deixar de considerar que o sentido de vida também é adquirido socialmente e que varia de uma cultura para outra. Em relação ao gênero, Prager (1996) e Reker e Wong (1988) encontraram mais semelhanças do que diferenças nas fontes de sentido entre homens e mulheres. No entanto, atividades criativas, relacionamento pessoal, servir aos outros, preservação de valores e ideais humanos e atividades religiosas foram mais frequentes entre as mulheres. Tais descobertas são importantes para a reflexão sobre a influência da cultura, da faixa etária e do gênero na atribuição de valor para os diversos aspectos da vida. Esses achados devem ser considerados nos delineamentos de pesquisas e nas conclusões sobre o que é importante para o viver.

Outros estudos também apontam os mesmos resultados dos estudos transculturais. Anchoo e Levi (1995) investigaram as fontes de sentido na vida entre idosos na faixa etária de 61 a 84 anos, e assim as situaram: relacionamento, altruísmo, criatividade, realização/status social, segurança, crescimento espiritual e aumento de posses materiais, atividades de lazer, preservação de valores e ideais humanos, sobrevivência, continuidade do self e geratividade, apreciação da natureza e das artes em geral. Ranst e Marcoen (1997) observaram que indivíduos mais velhos têm mais habilidades para perceber alguma perspectiva na vida, porque já viveram o bastante para avaliar no que efetivamente vale a pena investir, e se consideram mais realizadores de metas ou em processo de realização do que os mais jovens. No Brasil, Freire (2001) também encontrou esses dados. A explicação para tais resultados está associada à percepção de realização de tarefas evolutivas: os jovens ainda estão configurando suas uniões afetivas, consolidando-se profissionalmente, escolhendo e sedimentando suas escolhas. Os idosos já passaram por isso, e agora se voltam para tarefas que consideram mais significativas, nas quais valha a pena investir. A capacidade de perceber a situação e as capacidades realizadoras de modo mais realista auxilia no sucesso de persecução e efetivação das metas.

Somente estudos longitudinais poderiam dar informações mais precisas sobre como a maturidade e o processo de envelhecimento humano interagem, assim como sobre a constante necessidade de adaptação e ajuste para alterações no desenvolvimento e os esforços para manter estabilidade e continuidade no sentido de vida. Os estudos transversais, com diferentes grupos etários, retratam apenas momentos específicos da vida dos indivíduos, mas como têm menor exigência operacional, custo mais reduzido e demandam menos tempo, são mais utilizados. Há necessidade de dados longitudinais, mas isso não diminui a importância das pesquisas transversais.

Essas descobertas indicam direções sobre as fontes de sentido na vida, como elas se apresentam e como são percebidas na fase adulta. Também são de grande relevância para uma melhor compreensão da atribuição de significado ao longo da vida adulta e da velhice.

\section{Conclusão}

É crescente o número de estudos sobre o sentido de vida, ou seja, sobre o significado atribuído àquilo que é de grande valia para o viver, os quais fornecem importantes informações sobre pontos relevantes do processo de adaptação e de estímulo da vontade de viver. Esses dados contribuem enormemente para o estudo do desenvolvimento humano, para a compreensão da estabilidade e/ou mudança em fatores associados à adaptação e ao ajustamento - temas esses ligados à saúde geral. No processo de envelhecimento, cada vez mais os recursos internos de enfrentamento são requeridos, porque os indivíduos estão mais expostos a perdas de todos os tipos, as quais se constituem em fatores que interferem na vontade de viver.

Os dois principais grupos de pesquisas dão grandes contribuições para a Psicologia. Os estudos que definem o construto mostram como ele pode ser importante para a saúde, na medida em que apontam melhores resultados na recuperação de doenças, no enfrentamento de 
enfermidades de entes queridos e menores chances de adoecer. Em relação ao aporte para o estudo do desenvolvimento do adulto, as pesquisas apontam para a continuidade do sentido na vida e continuidade do self ao longo da vida. Quanto à pesquisa sobre as fontes de sentido na vida, a maior contribuição é relativa à descoberta dos pontos mais relevantes para os indivíduos na atribuição de significado à vida. Nessa perspectiva, os estudos transculturais dão pistas importantes sobre fatores que são comuns ao gênero e à faixa etária, como, por exemplo, o fato de as mulheres priorizarem conceitos humanistas, sociais e culturais. Já os homens valorizaram segurança financeira. Os homens mais velhos demonstraram interesse em áreas de sentido que transcendiam o self, enquanto os mais jovens priorizaram áreas que refletiam preocupação com o self, como experiências de crescimento e realização pessoal, deixar legado para as próximas gerações e participação em atividades hedonistas. Já satisfação de necessidades básicas, relações pessoais, preservação de valores e ideais humanos foram apontadas pela maioria dos indivíduos adultos, o que indica que algumas fontes de sentido podem ser comuns à espécie. Mas essas mesmas pesquisas alertam para a importância dos fatores relevantes para o viver e da cultura na atribuição de significado.

A dedicação dos pesquisadores ao tema aprimorou os instrumentos disponíveis, porém, ainda são necessários mais dados, de diferentes culturas e grupos etários, para que se possa compreender melhor os caminhos do desenvolvimento das fontes de sentido na vida e clarificar sua evolução e possíveis mudanças e/ou continuidade ao longo da vida. Nesse sentido, dados de estudos longitudinais e mais informações de pesquisas transculturais seriam de grande valia. Também é importante que os instrumentos de pesquisa sejam adaptados à realidade de cada país, uma vez que a cultura se apresenta como fator relevante.

Os dois grupos de pesquisas revelam uma grande possibilidade de continuidade na atribuição de importância de algumas fontes de significado. Revelam também a similaridade na escala de valores de alguns fatores, independentemente da cultura, o que permite concluir que componentes do sentido de vida são reconhecidos pelos indivíduos, independentemente de onde eles vivem, e fazem parte do desenvolvimento.

\section{Referências}

Anchoo, O., \& Levi, D. (1995). The use of focus group in determining sources of personal meaning for young-old and old-old Israelis. Ramat Aviv, Israel: Tel Aviv University.

Atchley, R. C. (1989). A continuity theory of normal aging. The Gerontologist, 29, 183-189.

Baumeister, R. F. (1991). Meanings of life. New York: The Guilford Press.

Calache, I. (1993). Sentido da vida: Uma questão de saúde mental do idoso. Dissertação de Mestrado não-publicada, Escola de Enfermagem, Universidade de São Paulo, Ribeirão Preto, SP.
Davis, C. G., Nolen-Hoeksema, S., \& Larson, J. (1998). Making sense of loss and benefiting from the experience: Two construal of meaning. Journal of Personality and Social Psychology, 75(2), 561-574.

De Vogler, K. L., \& Ebersole, P. (1980). Categorization of college students' meaning of life. Psychological Reports, 46, 387-390.

Diniz, S. A. (1992). Sentido da vida: Base para a compreensão do alcoolista. Dissertação de Mestrado não-publicada, Escola de Enfermagem, Universidade de São Paulo, Ribeirão Preto, SP.

Fiske, M., \& Chiriboga, D. A. (1991). Change and continuity in adult life. San Francisco, CA: Jossey-Bass.

Frankl, V. E. (1999). Psicoterapia e sentido de vida: Fundamentos da Logoterapia e análise existencial (3. ed.). São Paulo, SP: Quadrante.

Freire, S. A. (2001). Bem-estar subjetivo e metas de vida: Um estudo transversal com homens e mulheres pertencentes a três faixas de idade. Tese de Doutorado não-publicada, Faculdade de Educação, Universidade Estadual de Campinas, SP.

Fry, P. S. (1998). The development of personal meaning and wisdom in adolescence: A reexamination of moderating and consolidating factors and influences. In P. T. P. Wong \& P. S. Fry (Eds.), The human quest for meaning: A handbook of psychological research and clinical applications (pp. 91110). Mahwah, NJ: Lawrence Erlbaum.

Fry, P. S. (2001). The unique contribution of key existential factors to the prediction of psychological well-being of older adults following spousal loss. The Gerontologist, 41, 69-81.

Guttman, D., \& Huyck, M. H. (1994). Development and pathology in postparental men: A community study. In E. H. Thompson Jr. (Ed.), Older men's lives (pp. 104-121), Thousand Oaks, CA: Sage.

Halama, P. (2000). Dimension of life meaning as factors of coping. Studia Psychologica, 42, 339-350.

Huf, D. D. (1999). A assistência espiritual em Enfermagem na dimensão noética à luz da análise existencial de Viktor Frankl. Dissertação de Mestrado não-publicada, Escola de Enfermagem, Universidade de São Paulo, Ribeirão Preto, SP.

Kahana, E., \& Kahana, B. (2001). Successful aging among people with HIV/AIDS. Journal of Clinical Epidemiology, 54(Suppl.), S53-S56.

Kaufman, S. R. (1987). The ageless self: Sources of meaning in late life. Madison, WI: University of Wisconsin Press.

Kirby, S. E., Coleman, P. G., \& Daley, D. (2004). Spirituality and well-being in frail and nonfrail older adults. Journal of Gerontology: Psychological Sciences, 59B(3), 123-129.

Krause, N. (2003). Religious meaning and subjective wellbeing in later life. Journal of Gerontology: Social Sciences, $58 B(3)$, S160-S170.

Krause, N. (2004). Common facets of religion, unique facets of religion, and life satisfaction among older African Americans. The Journals of Gerontology Series B: Psychological Sciences and Social Sciences, 59(Suppl.), S109-S117.

Krause, N. (2007). Longitudinal study of social support and meaning in life. Psychology and Aging, 22(3), 456-469.

Lazarus, R. S. (1998). Coping with aging: Individuality as a key to understanding. In I. H. Nordhus, G. R. VandenBos, S. Berg, \& P. Fromholt (Eds.), Clinical Geropsychology (pp. 109-127). Washington, DC: American Psychological Association. 
Leonardi, J. (2007). O caminho noético: O canto e as danças circulares como veículos da saúde existencial no cuidar. Dissertação de Mestrado não-publicada, Escola de Enfermagem, Universidade de São Paulo, Ribeirão Preto, SP.

Prager, E. (1996). Exploring personal meaning in an agedifferentiated Australian sample: Another look at the sources of meaning profile (SOMP). Journal of Aging Studies, 10(2), 117-136.

Prager, E. (1997). Meaning in later life: An organizing theme for gerontological curriculum design. Educational Gerontology, 23, 1-13.

Ranst, N. V., \& Marcoen, A. (1997). Meaning in life of young and elderly adults: An examination of the factorial validity and invariance of the Life Regard Index. Personality and Individual Differences, 22(6), 877-884.

Reker, G. T. (1996). Manual of the Sources of Meaning ProfileRevised (SOMP-R). Procedures Manual: Research Edition. Ontario, Canada: Student Psychologists Press.

Reker, G. T. (1997). Personal meaning, optimism, and choice: Existential predictors of depression in community and institutional elderly. The Gerontologist, 37(6), 709-716.

Reker, G. T. (2000). Theoretical perspective, dimensions and measurement of existential meaning. In G. T. Reker \& K. Chamberlain (Eds.), Exploring existential meaning. Optimizing human development across the life span (pp. 3955). Thousand Oaks, CA: Sage.

Reker, G. T. (2001). Manual Life Attitude Profile - Revised. Ontario, Canada: Student Psychologists Press.

Reker, G. T., Peacock, E. J., \& Wong, P. T. P. (1987). Meaning and purpose in life and well-being: A life-span perspective. Journal of Gerontology, 42, 44-49.

Reker, G. T., \& Wong, P. T. P. (1988). Aging as an individual process: Toward a theory of personal meaning. In J. E. Birren \& V. L. Bengston (Eds.), Emergent theories of aging (pp. 214-246). New York: Springer.

Sommerhalder, C., \& Goldstein, L. L. (2006). O papel da religiosidade e da espiritualidade na vida adulta e na velhice. In E. V. Freitas, L. Py, F. A. X. Cançado, M. L. Gorzoni, \& A. L. Neri (Eds.), Tratado de Geriatria e Gerontologia (2 ed. rev., pp. 1307-1315). Rio de Janeiro, RJ: Guanabara Koogan.

Sommerhalder, C., \& Erbolato, R. M. P. L. (2010). Sentido de vida e relacionamentos significativos na velhice. In L. Araújo, C. Carvalho, \& V. Lucena, Diversidades do envelhecer: Uma abordagem multidisciplinar. Teresina, PI: Editora da Universidade Federal do Piauí.

Thompson, S. C., \& Janigiana, S. (1988). Life scheme: A framework for understanding the search for meaning. Journal of Social and Clinical Psychology, 7, 260-280.

Thurner, M. (1975). Continuities and discontinuities in value orientation. In M. F. Lowenthal, M. Thurner, \& D. Chiriboga (Eds.), Four stages of life: A comparative study of women and man facing transitions (pp. 176-200). San Francisco: Jossey-Bass.

Vitola, J. C. (1998). Terceira idade: Tendência atualizante e sentido de vida. Psico, 29(1), 63-88.

Wong, P. T. P. (1998). Spirituality, meaning and successful aging. In P. T. P. Wong \& P. S. Fry (Eds.), The human quest for meaning: A handbook of psychological research and clinical applications (pp. 395-435). Mahwah, NJ: Lawrence Erlbaum.
Zaleski, E. G. F. (1996). Sentido de vida do portador da síndrome da imunodeficiência adquirida: Uma questão de saúde mental para ações de Enfermagem. Dissertação de Mestrado não-publicada, Escola de Enfermagem, Universidade de São Paulo, Ribeirão Preto, SP.

Zaleski, E. G. F. (2001). A culpa existencial do portador de HIV/AIDS: Pesquisando e compreendendo seu significado. Tese de Doutorado não-publicada, Escola de Enfermagem, Universidade de São Paulo, Ribeirão Preto, SP.

Zika, S., \& Chamberlain, K. (1992). On the relation between meaning in life and psychological well being. British Journal of Psychology, 83, 133-145.
Recebido: 12/01/2009

$1^{a}$ revisão: $21 / 04 / 2009$ Aceite final: 16/06/2009 